\section{Progenitor cell therapy for epilepsy}

\section{By Tim Fulmer, Senior Writer}

University of California, San Francisco researchers have developed a cell therapy for epilepsy that aims to reduce the abnormal neuronal excitability that underlies the disease. ${ }^{1}$ The transplantation of neuronal precursor cells to specific regions of the brain could also avoid the side effects associated with epilepsy drugs, which target neuronal firing throughout the brain. The researchers have founded a company, Neurona Therapeutics Inc., to further develop the transplant strategy.

Epilepsy is characterized by excess neuronal excitation in regions of the brain that can lead to seizures and loss of consciousness. About one-third of epileptics develop resistance to the marketed drugs, which target neurotransmitter receptors and ion channels to prevent seizures. The therapeutics can also cause cognitive side effects such as fatigue, dizziness and slowed thinking processes.

The UCSF researchers previously found that certain types of embryonic neuronal precursor cells transplanted in the postnatal brains of mice were able to migrate throughout the cortex. In addition, the cells differentiated into functioning neurons that expressed $\gamma$-aminobutyric acid (GABA), the brain's primary inhibitory neurotransmitter. ${ }^{2}$

Based on that key finding, the team hypothesized that transplanting GABA-expressing neurons into the adult brain could increase inhibitory networks in regions of the cortex and help treat epilepsy.

They isolated neuronal precursors from the medial ganglionic eminence of mouse embryos and transplanted the cells into the cortex of 1- to 3-day-old mice. Immunostaining of CNS tissue, and electrophysiology studies, confirmed that the precursors dispersed throughout the cortex and differentiated into inhibitory neurons by day 30 .

The next step was trying the transplantation strategy in mice lacking the Kv1.1 potassium channel, a standard model of epilepsy.

In the Kv1.1 knockout mice, animals that had the same procedure at day 2 had lower duration and number of seizures than knockout mice that received mock control transplants. The transplants were also associated with nonsignificant increases in mean survival compared with control transplants ( 56 vs. 49 days; $p=0.16$ ).

The authors concluded that transplantation of the neuronal precursor cells "could potentially serve as a cell-based therapeutic approach for human epilepsy."

"This is the most comprehensive study of stem cell therapy for epilepsy to date," said Jack Parent, professor of neurology at the University of Michigan Medical Center and acting director of the university's epilepsy research program. “The next steps are to test the transplant approach in other rodent epilepsy models, eventually moving into larger animal models of the disease. In those models, it will be important to determine if any adverse effects are associated with the transplantation procedure."

Parent noted that other concerns relate to cell therapy more generally. "Besides the need for surgery and concerns that any progenitor cell therapy could give rise to tumorigenic cells, there's the potential problem of getting the transplanted cells to disperse widely in the brains of patients with multifocal epileptic seizure onsets," he said. "Moreover, if the transplanted cells do not survive long-term, the grafts may have to be repeated."

\section{Moving forward}

The UCSF researchers will continue to study the transplantation strategy in other models of epilepsy, said Scott Baraban, corresponding author and one of three principal investigators on the paper, which was published in the Proceedings of the National Academy of Sciences.

"A potential advantage of the GABA-producing cell transplants is that they locally and selectively inhibit excitation in the region of the engraftment and thus should be safer than small molecule inhibitors of neuronal excitation, which can influence neuronal firing throughout the brain," said Baraban, who is a professor of neurological surgery at UCSF.

Baraban said the transplantation strategy could help the nearly $30 \%$ of patients who develop resistance to epilepsy drugs. "We would not expect GABA resistance to develop since that molecule is an endogenous neurotransmitter and occurs throughout the CNS," he said.

Resistance to epilepsy drugs partially results from neuronal proteins that expel the drugs before they can have a therapeutic effect. ${ }^{3}$ That would be less likely to occur in the case of GABA, which is naturally expressed in many neurons and thus would not be targeted as a foreign molecule.

The three principal investigators on the PNAS article, along with Arnold Kriegstein, director of UCSF's Institute for Regeneration Medicine, have founded Neurona Therapeutics. The biotech is focused on the use of neuronal cell therapies to treat epilepsy and other neurological disorders such as Parkinson's disease (PD).

In addition to Baraban, the other UCSF investigators were Arturo Alvarez-Buylla, professor of neurological surgery, and John Rubenstein, professor of psychiatry and child psychiatry.

"In the longer term, we can envision generating transplantable 


\section{TARGETS \& MECHANISMS}

neuronal cells from iPS [induced pluripotent stem] cells or conventional stem cells. Moreover, those cells could potentially be engineered to produce not only GABA but other molecules that have a therapeutic effect in regions of the brain implicated in neurological disease," said Baraban.

Baraban and colleagues have applied for a patent covering the use of GABA-producing cells (interneurons) as a cell-based therapy to treat epilepsy and neurodegenerative diseases.

Fulmer, T. SciBX 2(35); doi:10.1038/scibx.2009.1330

Published online Sept. 10, 2009

\section{REFERENCES}

1. Baraban, S. et al. Proc. Natl. Acad. Sci. USA; published online Aug. 24, 2009; doi:10.1073/pnas.0900141106

Contact: Scott Baraban, University of California, San Francisco, Calif. e-mail: scott.baraban@ucsf.edu

2. Shetty, A. \& Hattiangady, B. Stem Cells 25, 2396-2407 (2007)

3. Sisodiya, S. et al. Brain 125, 22-31 (2002)

COMPANIES AND INSTITUTIONS MENTIONED

Neurona Therapeutics Inc., San Francisco, Calif.

University of California, San Francisco, Calif.

University of Michigan Medical Center, Ann Arbor, Mich. 